\title{
Diabetes mellitus en COVID-19: ¿factor de riesgo o factor pronóstico?
}

\section{Diabetes mellitus and COVID-19: Risk or prognostic factor?}

\author{
Builes-Montaño $C E^{1}$, Ramírez-Rincón $A^{2}$
}

${ }^{1}$ Grupo de Diabetes, Hospital Pablo Tobón Uribe. Profesor Asociado, Sección de Endocrinología y Metabolismo, Universidad de Antioquia. Miembro de número, Asociación Colombiana de Endocrinología, Diabetes y Metabolismo.

${ }^{2}$ Miembro de número, Asociación Colombiana de Endocrinología, Diabetes y Metabolismo. Clínica Integral de Diabetes (CLID). Profesor Titular, Universidad Pontifica Bolivariana.

Autor de correspondencia: Carlos E. Builes-Montaño Correo electrónico: cbuiles@hptu.org.co

\section{Resumen}

Antecedentes y propósito: la diabetes mellitus es una de las enfermedades crónicas no transmisibles de mayor prevalencia en el mundo. La frecuencia con la que se reporta en los pacientes con COVID-19 es alta. Sin embargo, no es claro si las personas que padecen diabetes mellitus tienen un mayor riesgo de infección o, si una vez infectados, tienen un peor pronóstico. Nuestro propósito fue revisar y analizar la información disponible de COVID-19 y diabetes mellitus e intentar entender mejor el riesgo al que están expuestas las personas con diabetes mellitus durante la pandemia por COVID-19. Métodos: se revisaron las bases de datos PubMed, Cochrane Database of Systematic Reviews, Google Scholar, Scopus y Epistemonikos en búsqueda de registros nacionales epidemiológicos y revisiones sistemáticas, utilizando los términos "Diabetes Mellitus" “COVID-19", "Factores de riesgo", "Pronostico", "Cuidado Critico", "Insuficiencia Respiratoria” y "Muerte”. Se seleccionaron para análisis las revisiones sistemáticas de las comorbilidades en pacientes con COVID-19, las que analizaban el curso de la enfermedad y los factores pronósticos en pacientes con COVID-19 y aquellas que incluían modelos de pronóstico. Resultados: la información disponible sugiere que la diabetes mellitus es una comorbilidad frecuente en las personas con COVID-19, pero es difícil diferenciar si esto es debido a la alta prevalencia de la diabetes mellitus o a un riesgo más alto de infección. Las personas con diabetes mellitus parecieran tener un riesgo más alto de presentar una forma grave o de morir a causa de la COVID-19.
Palabras clave: diabetes mellitus, COVID-19, factores de riesgo, pronóstico, cuidados críticos, insuficiencia respiratoria, muerte.

\section{Abstract}

Background and purpose: Diabetes mellitus is one of the most prevalent chronic non-communicable diseases in the world and it is frequently reported in patients with COVID-19. It is not clear if people with diabetes mellitus have a higher risk of infection or if once infected, they have a worse prognosis. Our purpose was to review and analyze the available information on COVID-19 and diabetes mellitus and try to better understand the risk to which people with diabetes mellitus are exposed during the COVID-19 pandemic. Methods: PubMed, Cochrane Database of Systematic Reviews, Google Scholar, Scopus, and Epistemonikos were reviewed for national epidemiological records and systematic reviews using the terms "Diabetes Mellitus", “COVID-19", "Risk Factors", "Prognosis", "Critical Care", "Respiratory Insufficiency" and "Death". Systematic reviews of comorbidities in patients with COVID-19, those that analyzed the course of the disease and prognostic factors in patients with COVID-19, and those that included prognostic models were selected for review. Results: The available information suggests that diabetes mellitus is a frequent comorbidity in people with COVID-19, but it is difficult to differentiate whether this is due to the high prevalence of diabetes mellitus or a higher risk of infection. People with diabetes mellitus appear to be at a greater risk of severe COVID-19 or dying from COVID-19.

Keywords: Diabetes mellitus, COVID-19, Risk factors, Prognosis, Critical care, Respiratory insufficiency, Death.

\section{Introducción}

Al momento de escribir esta revisión, se habían documentado más de 4 millones de casos en el mundo de la enfermedad infecciosa por coronavirus 2019 (COVID-19) y se tenía registro de cerca de 300.000 muertes $^{(1)}$. Reportes de varias partes 
del mundo sugieren que pudiera existir una asociación entre la COVID 19 y la diabetes mellitus (DM) y esta información proviene de dos fuentes, registros nacionales o regionales, en los cuales se incluyen pacientes de todo el espectro de gravedad de la enfermedad y reportes de pacientes que requirieron alguna forma de tratamiento hospitalario.

\section{Registros poblacionales}

Al interpretar los datos de registros, se debe tener en cuenta los posibles errores sistemáticos (sesgos) que pudieron afectar la información (Tabla 1).

Por tratarse de una enfermedad infecciosa nueva, diagnosticar adecuadamente a las personas que padecen la COVID 19 ha supuesto un gran reto para los sistemas de salud alrededor del mundo. El proceso diagnóstico está lleno de retos técnicos y logísticos ${ }^{(2)}$, y esto, combinado con la alta capacidad de propagación del virus, limita la cantidad de personas que pueden ser correctamente diagnosticadas. En Colombia, por ejemplo, a pesar del enorme esfuerzo del Gobierno nacional, las pruebas están restringidas a casos sospechosos que cumplan algunos criterios clínicos y epidemiológicos. Por otro lado, los registros de poblaciones son difíciles de llevar a cabo y, con frecuencia, tienen datos faltantes que suelen no ser aleatorios.

Estas restricciones hacen que los datos provenientes de registros de "pacientes diagnosticados" sean altamente susceptibles a sesgo de selección y, por lo tanto, las estimaciones (por ejemplo, incidencia de la COVID 19 o prevalencia de la DM) pudieran no ser confiables y difíciles de comparar.

El primer registro en una población de personas con COVID 19 fue reportado en China ${ }^{(3)}$. El gobierno chino decretó a la COVID 19 como una enfermedad de obligatorio reporte y para tal fin implementó una encuesta de donde se desprenden los datos de este registro. Para el registro de los casos, los pacientes clasificados como "sospechosos" eran aquellos con síntomas e historial de exposición, clínicamente diagnosticados, aquellos sospechosos que presentaban características de imágenes de neumonía y confirmados y aquellos casos sospechosos con resultados positivos de la prueba para detección de ARN del virus. Se reportaron 72.314 casos, 44.672 casos confirmados y se obtuvo información de comorbilidades en 20.812 de estos. La segunda comorbilidad más prevalente fue la DM (5,3\%), pero no es claro cómo se estableció el diagnóstico de las comorbilidades. De las 504 personas que murieron y de las cuales se tenía información sobre comorbilidades, en el $19,7 \%$ se reportó DM. Esta prevalencia de DM es casi la mitad a la reportada en el país ${ }^{(4)}$.

España ha sido uno de los países con más casos de COVID 19 registrados y también con uno de los registros de acceso público más detallado de los pacientes. Las comunidades autónomas notifican diariamente la información al Ministerio de Sanidad y, además, obtienen información clínica y epidemioló- gica de importancia. En el informe del 27 de abril de 2020 se incluía información de 198.876 pacientes de los 209.465 casos registrados en el país. De estos, la presencia o ausencia del antecedente de DM se pudo establecer en 110.111 y la prevalencia reportada fue $16,2 \%$ mayor a la reportada en la población general del país ${ }^{(4)}$. De los 52.903 casos en los cuales se conocía si había o no presentado neumonía, el 21\% de aquellos que tuvieron neumonía tenía antecedente de DM, comparado con el $10 \%$ de quienes no tuvieron neumonía; y de aquellos que requirieron hospitalización, el 25\% de quienes fueron internados en la unidad de cuidados intensivos (UCI) tenían DM, comparado con el $22 \%$ de aquellos que no ingresaron a la UCI (5).

Italia, el país europeo que mayor impacto ha tenido por la COVID 19, lleva un registro detallado de las personas que mueren a causa de la infección. En un reporte del 20 de marzo, que incluía 3200 registros, la edad promedio de las personas que murieron a causa de la COVID-19 fue de 78,5 años y la mayoría (70\%) eran hombres. El $34 \%$ de las personas que fallecieron tenían DM ${ }^{(6)}$

El Centro para el Control y Prevención de los Estados Unidos (CDC) reportó de forma preliminar las características de 7162 pacientes con datos disponibles de los 122.653 que habían sido diagnosticados por prueba para la detección del ARN del virus en ese país, entre el 12 de febrero y el 28 de marzo. El 10,9\% de estos pacientes tenían antecedente de DM, y fue mucho más común este antecedente entre aquellos hospitalizados (24\%) admitidos en una UCI (32\%), que en aquellos que recibieron un tratamiento ambulatorio $(6 \%){ }^{(7)}$.

\section{La diabetes mellitus como factor de riesgo para adquirir la COVID-19}

Un factor de riesgo es una característica que predispone a la aparición de una condición. Entonces, ¿las personas que tienen diabetes mellitus tienen una mayor probabilidad de padecer la COVID-19? Infortunadamente, es muy temprano, muy poco tiempo ha pasado desde el inicio de la enfermedad y no contamos con estudios longitudinales que permitan conocer la incidencia de la COVID-19 en las personas con diabetes mellitus. Toda la información que tenemos proviene de estudios de corte transversal o de casos y controles y la mayoría en personas hospitalizadas. Esto solo permite conocer la prevalencia de la diabetes mellitus en las personas con COVID-19.

Al haber sido China el primer país en ser afectado, es de esperarse que la mayoría de la información provenga de este país y esto puede hacer que los resultados de los estudios no sean generalizables. Varias revisiones sistemáticas (Tabla 2) han reportado una prevalencia entre el 6,2\% y el 11,9\% de diabetes mellitus entre las personas con COVID-19 ${ }^{(8-13)}$. La proporción hombre a mujer es cercana a 1:1 y la media o mediana de la edad está alrededor de los 50 años. La comorbilidad más frecuentemente reportada en todas las revisiones 
sistemáticas ha sido la hipertensión arterial, en cerca del 20\% de los pacientes. Todos los estudios incluidos fueron reportes de caso o estudios de corte transversal. Solo en un artículo se reporta la evaluación de la calidad de los estudios incluidos ${ }^{(10)}$ y estos eran de calidad intermedia o alta. La heterogeneidad fue baja en todos los reportes, al igual que el riesgo de sesgo de publicación.

\section{La diabetes mellitus como factor pronóstico en las personas con COVID-19}

Un factor pronóstico es una característica que predice la evolución de una enfermedad después de su inicio. Entonces, ¿las personas que tienen diabetes mellitus tienen un peor pronóstico de la COVID-19? Los datos para estimar este riesgo sufren de las mismas limitaciones discutidas previamente.

Tres revisiones sistemáticas han evaluado los factores tanto clínicos, como las pruebas de laboratorio que se asocian con una posibilidad de tener una forma grave de la COVID-19 (Tabla 3) ${ }^{(14-16)}$. Los resultados se basan en estudios de cohorte retrospectiva y la definición de gravedad se tomó de los estudios primarios, criterio en el que había gran heterogeneidad. Una publicación evaluó la asociación con el riesgo de morir ${ }^{(14)}$, otra, con el de tener una enfermedad grave ${ }^{(15)}$ y la otra combinó el desenlace en la posibilidad de tener una enfermedad crítica o de morir ${ }^{(16)}$. En todas las publicaciones, la diabetes mellitus se asoció con una mayor posibilidad de un peor pronóstico. La evaluación del riesgo de sesgo, a criterio de los autores, fue bajo, al igual que la heterogeneidad, y la calidad de los estudios primarios fue evaluada como moderada o alta.

Una revisión sistemática evaluó la asociación de solo las comorbilidades y el pronóstico de la COVID-19 (17). Se incluyeron 6 estudios de cohorte retrospectivos de alta calidad, de acuerdo con la clasificación de Ottawa. La gravedad de la enfermedad se definió según la necesidad de hospitalización en la UCI o los datos clínicos disponibles en los estudios, aunque estos criterios no fueron uniformes entre los estudios. La dia- betes mellitus se asoció con una mayor posibilidad de un peor pronóstico de la enfermedad, tanto en la población total del estudio (odds ratio [OR]: 2,47; IC 95\%: 1,67-3,66), como en el subgrupo de pacientes clasificados solo con base en los síntomas (OR: 2,66; IC 95\%: 1,73-4,10).

La incertidumbre alrededor del pronóstico de la COVID-19 ha impulsado la creación de múltiples modelos de predicción, tanto para el diagnóstico, como para el pronóstico. Sin embargo, la diabetes mellitus no hace parte de los factores incluidos con mayor frecuencia como predictor en los modelos ${ }^{(18)}$.

\section{Conclusión}

Los datos disponibles que asocian la COVID-19 y la diabetes mellitus deben analizarse en el contexto de las limitaciones (baja calidad) y los riesgos (alto de sesgo) de los estudios y reportes de los cuales provienen. La mayoría provienen de un solo país, China, por lo cual no es posible excluir la raza como un factor que pudiera influir en los resultados. La mayoría de los estudios son retrospectivos y tienen muchos datos faltantes; además, la exclusión de pacientes que no cumplían con los desenlaces pone en alto riesgo de sesgo de selección a estos estudios. Por los registros poblacionales, las series de caso y de corte transversal, pareciera que la asociación entre el riesgo de adquirir la COVID-19 y tener diabetes mellitus es el reflejo de un virus con una alta capacidad de contagio y una enfermedad altamente prevalente, ya que la prevalencia de diabetes mellitus en dichos estudios parece reflejar aquella de la población en la cual se presenta. Los estudios que han evaluado el pronóstico sugieren que aquellas personas con diabetes mellitus tienen un mayor riesgo de presentar una forma grave de la COVID-19 o de morir a causa de esta, aunque esta asociación pareciera ser menor a la que existe con otras comorbilidades, como la hipertensión arterial.

Como en muchas otras enfermedades infecciosas, las formas graves de la COVID-19 son el resultado de una compleja interacción entre el virus, los factores del huésped y el ambiente.

Tabla 1. Registros poblacionales

\begin{tabular}{|c|c|c|c|c|c|}
\hline Autor & $\begin{array}{c}\mathrm{N} \\
\text { (\% hombres) }\end{array}$ & Edad* & País & $\begin{array}{l}\text { Prevalencia de } \\
\text { DM en el reporte }\end{array}$ & $\begin{array}{l}\text { Prevalencia de } \\
\text { DM en el país(4) }\end{array}$ \\
\hline $\begin{array}{l}\text { Novel Coronavirus Pneumonia Emergency } \\
\text { Response Epidemiology Team }{ }^{(3)}\end{array}$ & $44.672(51,4)$ & $\begin{array}{c}\text { 30-69 años el } \\
77,8 \% \text { de los casos }\end{array}$ & China & $5,3 \%$ & $10,9(10,2-13,7)$ \\
\hline $\begin{array}{l}\text { Red Nacional de Vigilancia } \\
\text { Epidemiológica }{ }^{(5)}\end{array}$ & $110.111(44,7)$ & $61(46-78)$ & España & $16,2 \%$ & $10,5(8,4-14,6)$ \\
\hline Covid-19 Surveillance Group ${ }^{(6)}$ & $3200(30,6)$ & $78,5(73-85)$ & Italia & $33,9 \%^{+}$ & $8,3(7,7-9,4)$ \\
\hline $\begin{array}{l}\text { Centro para el Control y Prevención de En- } \\
\text { fermedades de los Estados Unidos (CDC) }{ }^{(7)}\end{array}$ & 7,162 (NR) & No reportado & EE.UU. & $10,9 \%$ & $13,3(11,4-15,3)$ \\
\hline
\end{tabular}


Tabla 2. Revisiones sistemáticas que reportan la prevalencia de la diabetes mellitus en personas con COVID-19 hospitalizadas

\begin{tabular}{|c|c|c|c|c|}
\hline Autor & N (\% hombres) & Edad $^{\dagger}$ & Prevalencia de DM & País de los reportes \\
\hline Yang y colaboradores ${ }^{(8)}$ & $1576(56,6 \%)$ & 49,6 (NR) & 9,7\% (IC 95\%: 7,2\%-12,2\%) & China \\
\hline Emami y colaboradores ${ }^{(9)}$ & 3402 (NR) & NR & 7,87\% (IC 95\%: 6,57\%-9,28\%) & China \\
\hline Fang y colaboradores ${ }^{(10)}$ & $3470(52,6 \%)$ & 17 meses a 92 años & $7,3 \%$ (NR) & China* \\
\hline Lin y colaboradores ${ }^{(11)}$ & $47.936(51,7 \%)$ & NR & $6,2 \%$ & China* \\
\hline Nasiri y colaboradores ${ }^{(12)}$ & 4789 (NR) & 49 (NR) & $10,8(8,3 \%-13,3 \%)$ & China* \\
\hline $\begin{array}{l}\text { Rodríguez-Morales y colabora- } \\
\text { dores }{ }^{(13)}\end{array}$ & $2874(55,9 \%)$ & $51,9(46,06-57,89)$ & 11,9\% (IC 95\%: 9,1\%-14,6\%) & China* \\
\hline
\end{tabular}

DM: diabetes mellitus; NR: no reportado.

†Edad en años, media o mediana (desviación estándar o rango intercuartílico).

*Incluyeron pacientes de fuera de China: Yang, 11 casos; Ling, 23 casos; Nasiri, 126 casos; Rodríguez-Morales, 15 casos.

Tabla 3. Revisiones sistemáticas que reportan la diabetes mellitus como factor pronóstico en personas con COVID-19

\begin{tabular}{l|c|c|}
\hline \multicolumn{1}{c|}{ Autor } & N & OR (IC 95\%) para enfermedad grave y/o muerte \\
\hline Parohan y colaboradores ${ }^{(14)}$ & 21.640 & $3,11(1,1-8,8)$ \\
\hline Zhao y colaboradores $^{(15)}$ & 53.000 & $2,49(1,82-3,4)$ \\
\hline Zheng y colaboradores $^{(16)}$ & 3027 & $3,68(2,68-5,03)$ \\
\hline Huang y colaboradores $^{(17)}$ & 1558 & $2,47(1,67-3,66)$ \\
\hline
\end{tabular}

\section{Conflicto de interés}

Los autores certifican que no tienen afiliaciones con o participación en cualquier organización o entidad con cualquier interés financiero (como honorarios, becas educativas, participación como oradores, empleo, consultorías, propiedad de acciones u otro interés patrimonial) o interés no financiero (como relaciones personales o profesionales, afiliaciones, conocimientos o creencias) en el tema o materiales discutidos en este manuscrito.

\section{Financiación}

Los autores declaran que no recibieron recursos para la elaboración de este manuscrito.

\section{Referencias}

1. World Health Organization. Coronavirus disease (COVID-2019) situation reports [Internet]. [consultado 29 de mayo de 2020]. Disponible en: https://www.who.int/emergencies/diseases/novel-coronavirus-2019/ situation-reports.

2. Cheng MP, Papenburg J, Desjardins M, Kanjilal S, Quach C, Libman M, et al. Diagnostic Testing for Severe Acute Respiratory Syndrome-Related Coronavirus-2: A Narrative Review. Ann Intern Med. 2020;M20-1301. doi: 10.7326/M20-1301.

3. Epidemiology Working Group for NCIP Epidemic Response, Chinese Center for Disease Control and Prevention. [The epidemiological characteristics of an outbreak of 2019 novel coronavirus diseases (COVID-19) in China]. Zhonghua Liu Xing Bing Xue Za Zhi. 2020;41(2):145-51. doi: 10.3760/cma. j.issn.0254-6450.2020.02.003.
4. Saeedi P, Petersohn I, Salpea P, Malanda B, Karuranga S, Unwin N, et al. Global and regional diabetes prevalence estimates for 2019 and projections for 2030 and 2045: Results from the International Diabetes Federation Diabetes Atlas, $9^{\text {th }}$ edition. Diabetes Res Clin Pract. 2019;157:107843. doi: 10.1016/j.diabres.2019.107843.

5. Red Nacional de Vigilancia Epidemiológica. Informe sobre la situación de COVID-19 en España. Informe COVID-19 n² 26. 27 de abril de 2020 [Interner]. Disponible en: https://bit.ly/3cdtzr8.

6. COVID-19 Surveillance Group I. Characteristics of COVID-19 patients dying in Italy: Report based on available data on March 20th, 2020 [Internet]. Disponible en: https://bit.ly/2TNE51W.

7. CDC COVID-19 Response Team. Preliminary estimates of the prevalence of selected underlying health conditions among patients with coronavirus 
disease 2019 -- United States, February 12-March 28, 2020. MMWR Morb Mortal Wkly Rep. 2020;69(13):382-6. doi: 10.15585/mmwrmm6913e2.

8. Yang J, Zheng Y, Gou X, Pu K, Chen Z, Guo Q, et al. Prevalence of comorbidities in the novel Wuhan coronavirus (COVID-19) infection: a systematic review and meta-analysis. Int J Infect Dis. 2020;94:91-5. doi: 10.1016/j. ijid.2020.03.017

9. Emami A, Javanmardi F, Pirbonyeh N, Akbari A. Prevalence of underlying diseases in hospitalized patients with COVID-19: a systematic review and meta-analysis. Arch Acad Emerg Med. 2020;8(1):e35.

10. Fang Z, Yi F, Wu K, Lai K, Sun X, Zhong N, et al. Clinical Characteristics of 2019 Coronavirus Pneumonia (COVID-19): An Updated Systematic Review [Internet]. medRxiv. 2020. Disponible en: https://bit.ly/2XFVyKK. doi: https://doi.org/10.1101/2020.03.07.20032573.

11. Yaqian M, Lin W, Wen J, Chen G. Clinical and pathological characteristics of 2019 novel coronavirus disease (COVID-19): a systematic review. medRxiv. 2020. Disponible en: https://bit.ly/2XbPBWI. doi: https://doi.org/10.1101 /2020.02.20.20025601.

12. Nasiri MI, Haddadi S, Tahvildari A, Farsi Y, Arbabi M, Hasanzadeh S, et al. COVID-19 clinical characteristics, and sex-specific risk of mortality: Systematic Review and Meta-analysis. medRxiv. 2020. Disponible en: https://bit. ly/3dgOQBh. doi: https://doi.org/10.1101/2020.03.24.20042903.
13. Rodriguez-Morales AJ, Cardona-Ospina JA, Gutiérrez-Ocampo E, VillamizarPeña R, Holguin-Rivera Y, Escalera-Antezana JP, et al. Clinical, laboratory and imaging features of COVID-19: A systematic review and meta-analysis. Travel Med Infect Dis. 2020;34:101623. doi: 10.1016/j.tmaid.2020.101623.

14. Parohan M, Yaghoubi S, Seraj A, Javanbakht MH, Sarraf P, Djalali M. Risk factors for mortality of adult inpatients with Coronavirus disease 2019 (COVID-19): a systematic review and meta-analysis of retrospective studies. medRxiv. 2020. Disponible en: https://bit.ly/2AfDgI9. doi: https://doi. org/10.1101/2020.04.09.20056291.

15. Zhao X, Zhang B, Li P, Ma C, Gu J, Hou P, et al. Incidence, clinical characteristics and prognostic factor of patients with COVID-19: a systematic review and meta-analysis. medRxiv. 2020. Disponible en: https://bit.ly/2XGJU23. doi: https://doi.org/10.1101/2020.03.17.20037572,

16. Zheng Z, Peng F, Xu B, Zhao J, Liu H, Peng J, et al. Risk factors of critical \& mortal COVID-19 cases: A systematic literature review and meta-analysis. Infect. 2020;S0163-4453(20)30234-6. doi: 10.1016/j.jinf.2020.04.021.

17. Wang B, Li R, Lu Z, Huang Y. Does comorbidity increase the risk of patients with COVID-19: evidence from meta-analysis. Aging. 2020;12(7):6049-57. doi: 10.18632 /aging.103000.

18. Wynants L, Van Calster B, Bonten MM, Collins GS, Debray TP, De Vos M, et al. Prediction models for diagnosis and prognosis of covid-19 infection: systematic review and critical appraisal. BMJ. 2020;369:m1328. doi: 10.1136/ bmj.m1328. 